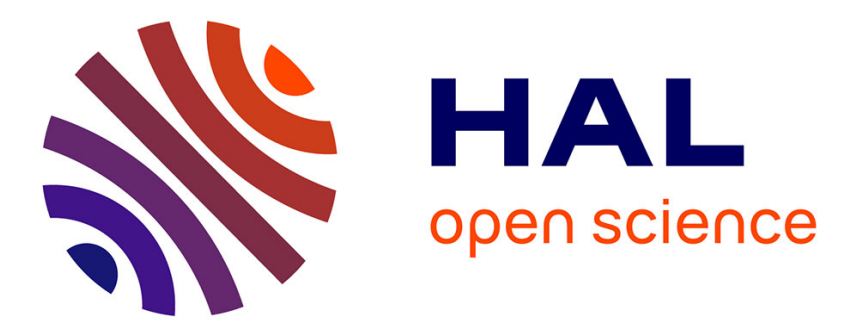

\title{
Control of linear systems with affine constraints: a gain-scheduling approach
}

\author{
Frédéric Grognard, Rodolphe Sepulchre, Georges Bastin
}

\section{To cite this version:}

Frédéric Grognard, Rodolphe Sepulchre, Georges Bastin. Control of linear systems with affine constraints: a gain-scheduling approach. 39th IEEE Conference on Decision and Control, Dec 2000, Sydney, Australia. pp.4785-4790, 10.1109/CDC.2001.914685 . hal-01091598

\section{HAL Id: hal-01091598 \\ https://hal.inria.fr/hal-01091598}

Submitted on 5 Dec 2014

HAL is a multi-disciplinary open access archive for the deposit and dissemination of scientific research documents, whether they are published or not. The documents may come from teaching and research institutions in France or abroad, or from public or private research centers.
L'archive ouverte pluridisciplinaire HAL, est destinée au dépôt et à la diffusion de documents scientifiques de niveau recherche, publiés ou non, émanant des établissements d'enseignement et de recherche français ou étrangers, des laboratoires publics ou privés. 


\title{
Control of linear systems with affine constraints: a gain-scheduling approach
}

\author{
F. Grognard ${ }^{\dagger}$, R. Sepulchre ${ }^{\ddagger}$, G. Bastin ${ }^{\dagger}$ \\ $\dagger$ Center for Systems Engineering and Applied Mechanics \\ Université catholique de Louvain, \\ Av. G. Lemaitre 4, B1348 Louvain-La-Neuve, Belgium. \\ Phone: +32-10-478030 Fax: +32-10-472180 \\ email:grognard@auto.ucl.ac.be \\ Institut Montefiore, B28 \\ Université de Liège, \\ B4000 Liège Sart-Tilman, Belgium. \\ email: r.sepulchre@ulg.ac.be
}

Submitted to the $39^{\text {th }}$ IEEE Conference on Decision and Control, Sydney, December 2000

\begin{abstract}
A gain scheduling based on a one-parameter family of Lyapunov functions is presented for the control of linear systems with affine constraints. The tuning of the parameter in the control law is assumed to result from a trade-off between the size of the state-space domain where the constraints are satisfied and the closed-loop performance. A target controller is chosen for local performance in this family. The proposed online scheduling is aimed at reaching the target controller in the fastest possible way, while guaranteeing satisfaction of the constraints along closed-loop solutions.
\end{abstract}




\section{Introduction}

This paper addresses the feedback control design of linear systems

$$
\dot{x}=A x+B u \quad x \in \mathbb{R}^{n}, u \in \mathbb{R}
$$

subject to $\mathrm{p}$ affine constraints

$$
L x+M u \leq N
$$

with $L \in \mathbb{R}^{p \times n}, M \in \mathbb{R}^{p}, N \in \mathbb{R}^{p}$.

Even though our primary concern will be the control of linear systems under input magnitude constraints (as in $[3,4,7]$ ) or magnitude and rate constraints $([2,6])$, the proposed method is, in principle, more general.

The realistic assumption that we adopt as a starting point is that the constraints (1.2) are not active locally, that is, any smooth stabilizing feedback satisfies the constraints in a neighborhood of the origin $x=0$.

Assumption 1 If $A+B K$ is an Hurwitz matrix, then $L x+M K x \leq N$ is satisfied for all $x$ in a neighborhood of the origin.

A stronger assumption adopted throughout the paper is that the constraints (1.2) can be satisfied with linear controllers in large regions of the state-space, at the expense of degraded performance. For instance, this is the well-known situation encountered when low-gain designs are used to address magnitude and rate limitations on the actuators.

Choosing an initial controller that ensures a sufficiently large region of attraction and a final controller that ensures good local performance, our objective is to design a scheduling ensuring the best possible transition between these two extreme controllers along the closed-loop solutions.

To this end, we will assume that our "initial" and "final" controllers belong to a one-parameter family of linear controllers:

$$
J u=-K(\lambda) x
$$

which, for each $\lambda \in(0,1]$ ensures the decrease of a quadratic Lyapunov function

$$
V(x, \lambda)=x^{T} P(\lambda) x
$$

along the solutions of (1.1) in the absence of the constraints (1.2) (with $K(\lambda)$ and $P(\lambda)>0$ continuously differentiable). The role of the parameter $J$ in (1.3) will be explained in Section 2. The construction of the one-parameter family (1.3) and (1.4) is not addressed in general in this paper, but an efficient 
procedure is proposed in [4] based on a parameterized Riccati inequality. More specific choices will be discussed in the applications section.

As a convention, the value $\lambda=1$ will correspond to the target controller that yields good local performance. However, we will be interested in dealing with initial conditions $x_{0}$ that initially force a smaller value of $\lambda$ in order to satisfy the constraints (1.2). Our gain-scheduling design will show how to adapt the parameter $\lambda$ along the closed-loop solutions such as to guarantee the fastest possible transition to the target controller while satisfying the constraints and ensuring convergence to the origin. This approach was first proposed in [4] in the restricted framework of magnitude constraints, with a Riccati-based family of Lyapunov functions (1.4) and controllers $u=-B^{T} P(\lambda) x$. A different family of controllers was recently proposed in [1], aimed at the online invariance of condition $B^{T} P(\lambda) x=0$.

The present paper generalizes the results of [4] and [1] in a unified framework. The generalization of magnitude input constraints to arbitrary affine constraints (1.2) is not merely notational. It is exemplified in the present paper by a new and successful design scheme for the control of linear systems under both magnitude and rate limitations on the control variable.

The paper is organized as follows: Section 2 discusses two different choices of controllers for a fixed family of Lyapunov functions. Section 3 describes the gain-scheduling algorithm itself. Section 4 addresses the particular case of input magnitude constraints, summarizing the results of [4] and [1] in the unified framework of this paper. Section 5 addresses the control of linear systems with input magnitude and rate constraints and different solutions are illustrated on the double integrator system.

\section{Controller gain}

The parameter $J$ is introduced in (1.3) to distinguish between explicit control laws $u=-K(\lambda) x(J=1)$ and implicit control laws specified by the invariance condition $K(\lambda) x=0(J=0)$. The reason why this distinction is rather important for the proposed gain-scheduling is now briefly explained.

By assumption, any fixed controller (1.3) will satisfy the constraints (1.2) in a neighborhood of the origin. A Lyapunov estimate of this region is given by the minimum level set $\bar{V}(\lambda)$ where one of the constraints becomes active.

The online requirement $V(x, \lambda) \leq \bar{V}(\lambda)$ will guarantee closed-loop convergence to the origin, but it is the main source of conservatism in the adaptation of $\lambda$. 
In the case $J=1, \bar{V}(\lambda)$ is computed as

$$
\begin{aligned}
\bar{V}(\lambda)=\min _{x \in \mathbb{R}^{n}} & x^{T} P(\lambda) x \\
& \text { s.t. } \exists i:\left(L_{i}-M_{i} K(\lambda)\right) x=N_{i}
\end{aligned}
$$

In the implicit case $J=0$, we assume the relative degree one condition $K(\lambda) B \neq 0$, so that, when $\lambda$ is fixed, the invariance condition $K(\lambda) x=0$ is ensured by the control law $u=-\frac{K(\lambda) A x}{K(\lambda) B} \cdot \bar{V}(\lambda)$ is then computed as

$$
\begin{aligned}
& \bar{V}(\lambda)=\min _{x \in \mathbb{R}^{n}} x^{T} P(\lambda) x \\
& \text { s.t. }\left\{\begin{array}{l}
\exists i:\left(L_{i}-M_{i} \frac{K(\lambda) A}{K(\lambda) B}\right) x=N_{i} \\
K(\lambda) x=0
\end{array}\right.
\end{aligned}
$$

Both in the explicit and implicit cases, $\bar{V}(\lambda)$ results from the minimization of the quadratic function under affine constraints. Presumably, the additional equality constraint in (2.6) will result in a larger value $\bar{V}(\lambda)$, thereby reducing the conservatism of the Lyapunov estimate.

The specification of the controller through the implicit relation $K(\lambda) x=0$ may of course pose a problem for the initialization of the control scheme. If no $\lambda_{0}$ exists such that $K\left(\lambda_{0}\right) x_{0}=0$, an initial phase of the control algorithm is necessary to bring the solution in an admissible region of the state-space. This part of the algorithm is somewhat decoupled from the gain-scheduling problem addressed here and will not be further discussed in the present paper. It is discussed in [1] in analogy with a sliding mode control approach where $K(\lambda) x=0$ would be the sliding surface and a "reaching mode" is necessary for initial conditions that do not belong to the sliding surface. In the sequel, the gain-scheduling will be called "explicit" in the case $J=1$ and "implicit" in the case $J=0$.

\section{Gain-scheduling}

Consider the feasibility region $\Gamma$ determined by

$$
\Gamma=\left\{(x, \lambda) \in \mathbb{R}^{n} \times(0,1] \mid x^{T} P(\lambda) x \leq \bar{V}(\lambda)\right\}
$$

By definition of $\bar{V}(\lambda)$ the fixed parameter controller $J u+K\left(\lambda_{0}\right) x=0$ yields closed-loop convergence without constraint violation for any initial condition $x_{0}$ such that $\left(x_{0}, \lambda_{0}\right) \in \Gamma$. Our gain-scheduling algorithm will determine an adaptation rule $\dot{\lambda} \geq 0$ and the accompanying control law such as to maximize $\dot{\lambda}$ along the closed-loop solutions and satisfy the constraints (1.2), while ensuring the closed-loop invariance of $\Gamma$. Invariance of $\Gamma$ will imply 
that the adaptation can be stopped at any time, the convergence of $x(t)$ to the origin being then guaranteed by the preceding argument.

In the explicit case $u=-K(\lambda) x$, invariance of the feasible region $\Gamma$ guarantees that the constraints are satisfied along the closed-loop solutions because the definition of $\bar{V}(\lambda)$ implies that $L x-M K(\lambda) x \leq N$ when $(x, \lambda) \in$ $\Gamma$.

Invariance of $\Gamma$ and satisfaction of the constraints is then guaranteed by the feedback rule:

$$
\lambda(x(t))=\max \{\eta \in(0,1]: V(x, \eta) \leq \bar{V}(\eta)\}
$$

or through the adaptation rule

$$
\begin{array}{ll}
\max & \dot{\lambda} \text { s.t. } \\
& \frac{d}{d t}(V(x, \lambda)-\bar{V}(\lambda))_{+} \leq 0 \quad \text { if } V(x, \lambda)=\bar{V}(\lambda)
\end{array}
$$

The feedback rule (3.7) was proposed by Megretski [4] in the particular case of the input magnitude constraints while (3.8) will be used for comparison with the implicit gain-scheduling developed below.

Rewriting the differential constraint in (3.8) as

$$
\frac{\partial V}{\partial x} \dot{x}+\left(\frac{\partial V}{\partial \lambda}-\left(\frac{\partial \bar{V}}{\partial \lambda}\right)_{+}\right) \dot{\lambda} \leq 0
$$

we see that, for an initial condition $\left(x_{0}, \lambda_{0}\right)$ satisfying $V\left(x_{0}, \lambda_{0}\right)=\bar{V}\left(\lambda_{0}\right)$, the adaptation rule is uniquely determined as

$$
\dot{\lambda}=-\left(\frac{\partial V}{\partial \lambda}-\left(\frac{\partial \bar{V}}{\partial \lambda}\right)_{+}\right)^{-1} \frac{\partial V}{\partial x} \dot{x}
$$

under the monotonicity assumption

$$
\frac{\partial V(x, \lambda)}{\partial \lambda}-\left(\frac{\partial \bar{V}(\lambda)}{\partial \lambda}\right)_{+}>0
$$

Assumption (3.10) guarantees a continuous evolution of $\lambda(t)$, in which case the feedback rule (3.7) is just the integral form of the adaptation rule (3.8) and expresses that the closed-loop solution $(x(t), \lambda(t))$ will stay on the boundary of $\Gamma$ until the target $\lambda_{f}=1$ is reached.

It is worthwhile noting that, even in the absence of the monotonicity condition (3.10), both the feedback rule (3.7) and the adaptation rule (3.8) guarantee a monotonic evolution of $\lambda(t)$. This is because $\dot{\lambda}=0$ is a feasible solution of (3.8) at any point of $\Gamma$. 
The feedback rule (3.7) is no longer valid in the case of an implicit gain-scheduling $J=0$. The control law enforcing the invariance condition $K(\lambda) x=0$ is given by

$$
u=\frac{1}{K(\lambda) B}\left(-K(\lambda) A x-\frac{\partial K}{\partial \lambda} x \dot{\lambda}\right)
$$

Because of the additional term $\frac{\partial K}{\partial \lambda} x \dot{\lambda}$ in (3.11), the constraints are no longer guaranteed to be satisfied when $(x, \lambda) \in \Gamma$.

To ensure closed-loop invariance of $\Gamma$ and satisfaction of the constraints, the adaptation rule $\dot{\lambda}$ must now be determined as the solution of the pointwise maximization

$$
\begin{array}{ll}
\max & \dot{\lambda} \text { s.t. } \\
& \frac{d}{d t}(V(x, \lambda)-\bar{V}(\lambda)) \leq 0 \quad \text { if } V(x, \lambda)=\bar{V}(\lambda) \\
& L x+\frac{M}{K(\lambda) B}\left(-K(\lambda) A x-\frac{\partial K}{\partial \lambda} x \dot{\lambda}\right) \leq N \\
& \dot{\lambda} \leq \dot{\lambda}_{\max }
\end{array}
$$

It must again be emphasized that the solution $\dot{\lambda}=0$ is feasible at any point of $\Gamma$, which ensures that the solution of (3.12) is non negative. The bound $\dot{\lambda}_{\max }(>0)$ is arbitrary, but prevents jumps in the evolution of $\lambda(t)$ and guarantees that the control (3.11) is well-defined.

Under normal circumstances, both the explicit and implicit gain schedulings just described will allow the parameter $\lambda$ to converge in finite time to the target $\lambda_{f}=1$, eventually leading to closed-loop convergence of the solution $x(t)$ with the fixed controller $J u+K(1) x=0$.

Alternatively, it may happen that $\lambda$ never reaches 1 , but converges to some $\bar{\lambda} \leq 1$. The next theorem guarantees closed-loop convergence of $x(t)$ to the origin in all cases.

Theorem 1 Consider a family of Lyapunov functions

$$
V(x, \lambda)=x^{T} P(\lambda) x \quad \lambda \in(0,1] \quad P(\lambda)>0
$$

whose time derivative, with $\lambda$ fixed, along the solutions of the linear system $\dot{x}=A x+B u$ is rendered negative definite by the explicit control law $u=$ $-K(\lambda) x$ or through the invariance condition $K(\lambda) x=0$. Then, the feedback rule (3.7) with

$$
u=-K(\lambda) x
$$

guarantees finite-time convergence of $\lambda(t)$ to 1 and convergence of $x(t)$ to the origin for any solution with initial condition $\left(x_{0}, \lambda_{0}\right)$ satisfying $V\left(x_{0}, \lambda_{0}\right) \leq$ $\bar{V}\left(\lambda_{0}\right)$. Likewise, the adaptation rule (3.12) with

$$
u=\frac{1}{K(\lambda) B}\left(-K(\lambda) A x-\frac{\partial K}{\partial \lambda} x \dot{\lambda}\right)
$$


guarantees convergence to the origin of $x(t)$ for any initial condition $\left(x_{0}, \lambda_{0}\right)$ satisfying $V\left(x_{0}, \lambda_{0}\right) \leq \bar{V}\left(\lambda_{0}\right)$ and $K\left(\lambda_{0}\right) x_{0}=0$.

Proof Closed-loop convergence of the solution $x(t)$ with the fixed controller $J u+K(1) x=0$ is guaranteed in both cases once the parameter reaches 1 and the adaptation is stopped.

Let us suppose that $\lambda(t)$ does not reach the target value $\lambda_{f}=1$ in finite time. $\lambda(t)$ is then an increasing function with an upper bound; therefore, there exists $\bar{\lambda} \leq 1$ such that $\lambda(t)$ converges to $\bar{\lambda} \leq 1$ as $t \rightarrow \infty$. The derivative of the Lyapunov function $V(x, \lambda)$ is:

$$
\dot{V}(x, \lambda)=x^{T} P(\lambda) A x+x^{T} A^{T} P(\lambda) x+2 x^{T} P(\lambda) B u+x^{T} \frac{d P(\lambda)}{d \lambda} x \dot{\lambda}
$$

with $u$ satisfying (3.13) or (3.14). In both cases, one has

$$
\dot{V}(x, \lambda)=-x^{T} R(\lambda) x+x^{T} S(\lambda) x \dot{\lambda}
$$

with $R(\lambda)>0$. Continuity of $S(\lambda)$ on $\left[\lambda_{0}, \bar{\lambda}\right]$ implies that one can find $\alpha>0$ such that $x^{T} S(\lambda) x \leq \alpha V(x, \lambda)$, which implies

$$
\dot{V}(x, \lambda) \leq \alpha \dot{\lambda} V(x, \lambda)
$$

and

$$
V(x(t), \lambda(t)) \leq V\left(x_{0}, \lambda_{0}\right) e^{\int_{0}^{t} \alpha \dot{\lambda}(\tau) d \tau}
$$

One deduces the upperbound $V(x(t), \lambda(t)) \leq V\left(x_{0}, \lambda_{0}\right) e^{\alpha\left(\bar{\lambda}-\lambda_{0}\right)}$ for all $t \geq$ 0 , which guarantees boundedness of $x(t)$ along the solutions. Let us now suppose that $x(t)$ does not converge to $x=0$ as $t \rightarrow \infty$. Integrating (3.15) yields

$$
V(x(t), \lambda(t))=-\int_{0}^{t} x(\tau)^{T} R(\lambda(\tau)) x(\tau) d \tau+\int_{0}^{t} x(\tau)^{T} S(\lambda(\tau)) x(\tau) \dot{\lambda}(\tau) d \tau
$$

Taking the limit for $t \rightarrow \infty$, the first term diverges to $-\infty$ and the second term is finite (because $x^{T} S(\lambda) x \leq \alpha V\left(x_{0}, \lambda_{0}\right) e^{\alpha\left(\bar{\lambda}-\lambda_{0}\right)}$ and $\int_{0}^{+\infty} \dot{\lambda}(\tau) d \tau=$ $\left.\bar{\lambda}-\lambda_{0}\right)$. This is in contradiction with the positiveness of $V$. We conclude that $x(t)$ converges to the origin, even when $\lambda(t)$ does not reach 1 in finite time.

In the explicit case $u=-K(\lambda) x$, if $\lambda(t)$ does not reach 1 in finite time, the convergence of $x(t)$ to the origin ensures that the solution $x(t)$ enters the set $\{x \mid V(x, 1) \leq \bar{V}(1)\}$ in finite time. Inside this set, the feedback rule (3.7) ensures $\lambda=1$, which is a contradiction. Therefore $\lambda(t)$ reaches 1 in finite time in the explicit case. 


\section{Input magnitude constraint}

The construction of a one-parameter family of Lyapunov functions is classical for linear systems $\dot{x}=A x+B u$ subject to the input constraint

$$
|u| \leq u_{\max }
$$

Assuming null controllability of the pair $(A, B)$, several authors have proposed the quadratic family $V(x, \lambda)=x^{T} P(\lambda) x$ generated from the Riccati equation

$$
P(\lambda) A+A^{T} P(\lambda)-P(\lambda) B B^{T} P(\lambda)=-Q(\lambda), \quad \lambda \in(0, \infty)
$$

with $Q(\lambda)>0$ and $\frac{d Q}{d \lambda}>0$ (see for instance $[3,7]$ ).

The choice $u=-B^{T} P(\lambda) x$ corresponds to an explicit specification of the control law $(J=1)$ and leads to the gain-scheduling algorithm proposed by Megretski in [4]. The solution obtained in [4] is

$$
\bar{V}(\lambda)=\frac{u_{\max }^{2}}{B^{T} P(\lambda) B}
$$

and

$$
\lambda(x)=\max \{\eta \in(0,1]: V(x, \eta) \leq \bar{V}(\eta)\}
$$

which indeed corresponds to the solution of (3.9) thanks to the monotonicity condition $\frac{\partial P}{\partial \lambda}>0$ and $\frac{\partial \bar{V}}{\partial \lambda}<0$

The implicit specification of the control law through the invariance condition $B^{T} P(\lambda) x=0(J=0)$ leads to the gain-scheduling proposed in our earlier work [1]. The maximal admissible level set for a given $\lambda>0$ is shown to be

$$
\bar{V}(\lambda)=\frac{u_{\max }^{2}\left(B^{T} P(\lambda) B\right)^{3}}{\left(B^{T} P(\lambda) A P(\lambda)^{-1} A^{T} P(\lambda) B\right)\left(B^{T} P(\lambda) B\right)-\left(B^{T} P(\lambda) A B\right)^{2}}
$$

For initial conditions that cannot satisfy $B^{T} P(\lambda) x_{0}=0$ for some $\lambda$, a reaching phase is first implemented by the control

$$
u=-\operatorname{sign}\left(B^{T} P\left(\lambda_{0}\right) x\right)
$$

with $\lambda_{0}$ small and fixed, which forces the convergence of the solution in finite time to a region of the state space where the constraints $B^{T} P\left(\lambda_{0}\right) x=0$ and $V\left(x, \lambda_{0}\right) \leq \bar{V}\left(\lambda_{0}\right)$ are satisfied.

Then the control $u$ and the adaptation rule are directly obtained from the pointwise optimization problem (3.12).

Comparisons of the two gain-scheduled algorithms on the double and triple integrators suggest that the implicit gain-scheduling usually results in faster convergence of the closed-loop solutions [1]. 


\section{$5 \quad$ Input magnitude and rate constraints}

Adding the rate constraint

$$
|\dot{u}| \leq \dot{u}_{\max }
$$

to the input constraint considered in the previous section, we need to construct a one-parameter family of Lyapunov functions for the extended statespace model

$$
\left\{\begin{array}{l}
\dot{x}=A x+B u \\
\dot{u}=v
\end{array}\right.
$$

A simple choice, that relies on the construction in the previous section, for the Lyapunov function is the "backstepping" augmentation [5]

$$
V((x, u), \lambda)=x^{T} P(\lambda) x+\left(u+B^{T} P(\lambda) x\right)^{2}
$$

A family of controllers

$$
J v=-K(\lambda)\left(\begin{array}{l}
x \\
u
\end{array}\right)
$$

must be constructed such that the time derivative

$$
\begin{aligned}
\dot{V}=x^{T} P A x+ & x^{T} A^{T} P x+2 x^{T} P B u \\
& +2\left(u+B^{T} P x\right)\left(v+B^{T} P A x+B^{T} P B u\right) \\
=-x^{T} Q x- & x^{T} P B B^{T} P x \\
& \quad+2\left(u+B^{T} P x\right)\left(v+B^{T} P A x+B^{T} P B u+B^{T} P x\right)
\end{aligned}
$$

is rendered negative. This is accomplished with the explicit controller $(J=1)$

$$
\begin{aligned}
v & =-K(\lambda)\left(\begin{array}{l}
x \\
u
\end{array}\right) \\
& =-\left(B^{T} P A x+B^{T} P B u+B^{T} P x+k\left(u+B^{T} P x\right)\right) \quad k>0(
\end{aligned}
$$

or through the implicit specification $(J=0)$

$$
u+B^{T} P(\lambda) x=0
$$

which corresponds to $K(\lambda)\left(\begin{array}{l}x \\ u\end{array}\right)=0$ for the limit case $k=+\infty$. For the explicit gain-scheduling based on (5.17), one obtains

$$
\lambda(x(t))=\max \{\eta \in(0,1]: V(x, \eta) \leq \bar{V}(\eta)\}
$$


where

$$
\bar{V}(\lambda)=\min \left(\bar{V}_{1}(\lambda), \bar{V}_{2}(\lambda)\right)
$$

with $\bar{V}_{1}$ corresponding to the minimal level set inside which the magnitude constraint is satisfied, and $\bar{V}_{2}$ corresponding to the minimal level set inside which the rate constraint is satisfied:

$$
\begin{aligned}
\bar{V}_{1}(\lambda) & =\min _{x} V\left(\left(x, u_{\max }\right), \lambda\right) \\
\bar{V}_{2}(\lambda) & =\min _{(x, u)} V((x, u), \lambda) \\
& \text { s.t. } K(\lambda)\left(\begin{array}{l}
x \\
u
\end{array}\right)=\dot{u}_{\max }
\end{aligned}
$$

The implicit gain-scheduling (5.18) requires no "reaching phase" if one assumes that the initial control variable $u$ can be freely initialized at the value $-B^{T} P(\lambda) x$.

For $\lambda>0$ fixed, invariance of the manifold $u=-B^{T} P(\lambda) x$ then imposes

$$
\begin{aligned}
v & =-B^{T} P(\lambda) A x-B^{T} P(\lambda) B u \\
& =-B^{T} P(\lambda)\left(A-B B^{T} P(\lambda)\right) x=G^{T}(\lambda) x
\end{aligned}
$$

so that $\bar{V}_{1}$ and $\bar{V}_{2}$ are now replaced by

$$
\begin{array}{r}
\bar{V}_{1}(\lambda)=\min _{(x, u)} x^{T} P(\lambda) x+\left(u+B^{T} P(\lambda) x\right)^{2} \\
\text { s.t. }\left\{\begin{array}{l}
u+B^{T} P(\lambda) x=0 \\
u=u_{\max }
\end{array}\right.
\end{array}
$$

which reduces to

$$
\begin{aligned}
\bar{V}_{1}(\lambda)=\min _{x} x^{T} P(\lambda) x \\
\text { s.t. } B^{T} P(\lambda) x=u_{\max }
\end{aligned}
$$

and, similarly

$$
\begin{aligned}
& \bar{V}_{2}(\lambda)=\min _{x} x^{T} P(\lambda) x \\
& \text { s.t. } G^{T}(\lambda) x=\dot{u}_{\max }
\end{aligned}
$$

whose solutions are $\bar{V}_{1}(\lambda)=\frac{u_{\max }^{2}}{B^{T} P(\lambda) B}$ and $\bar{V}_{2}(\lambda)=\frac{v_{\max }^{2}}{G^{T}(\lambda) P^{-1}(\lambda) G(\lambda)}$.

The control $v$ and the adaptation rule $\dot{\lambda}$ are then obtained from the pointwise optimization (3.12).

The application of these adaptation schemes are now illustrated on a simple example. 
Example On Figure 2, we compare the efficiency of different algorithms for the control of the double integrator with control rate and amplitude constraints:

$$
\left\{\begin{array}{l}
\dot{x}_{1}=x_{2} \\
\dot{x}_{2}=u
\end{array} \quad|u| \leq 1,|\dot{u}| \leq 1\right.
$$

with the extension $\dot{u}=v$. Solving the Riccati equation (4.16) with:

$$
Q(\lambda)=\left(\begin{array}{cc}
\lambda^{4} & 0 \\
0 & \lambda^{2}
\end{array}\right)
$$

results in the Lyapunov matrix:

$$
P(\lambda)=\left(\begin{array}{cc}
\sqrt{3} \lambda^{3} & \lambda^{2} \\
\lambda^{2} & \sqrt{3} \lambda
\end{array}\right)
$$

and the family of low-gain controls is then:

$$
u=-B^{T} P(\lambda) x=-\lambda^{2} x_{1}-\sqrt{3} \lambda x_{2} \quad \lambda>0
$$

which is a typical low-gain control for second order systems. We arbitrarily consider that the target behavior of the closed-loop system is for $\lambda=1$.

In this case, the explicit controller (with $J=1$ and $k=1$ ) is

$$
\begin{aligned}
v & =-B^{T} P A x-B^{T} P B u-B^{T} P x-\left(B^{T} P x+u\right) \\
& =-\lambda^{2}\left(x_{2}+2 x_{1}\right)-\sqrt{3} \lambda\left(u+2 x_{2}\right)-u
\end{aligned}
$$

and the implicit controller specification $(J=0$ and $k=+\infty)$ yields

$$
u+\lambda^{2} x_{1}+\sqrt{3} \lambda x_{2}=0
$$

$\bar{V}(\lambda)$ is calculated and drawn on Figure 1 both for the explicit and the implicit case. We see that, for all $\lambda$ between 0 and $1, \bar{V}$ is larger for the implicit scheduling. This explains why we expect faster convergence with the implicit gain-scheduling.

This expectation is confirmed by the the simulations; three controllers are compared:

(i) a fixed low-gain explicit controller (dotted line curves);

(ii) a gain scheduling based on the explicit controller (dash-dotted line curves);

(iii) a gain-scheduling based on the implicit controller (solid line curves). 


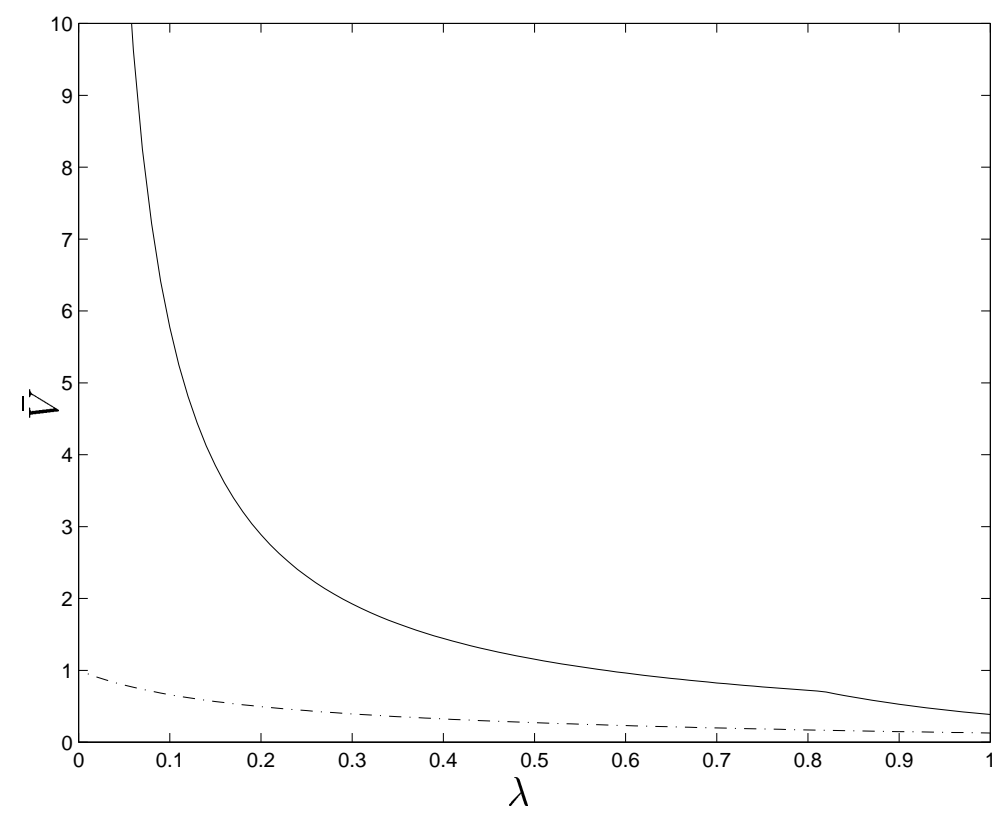

Figure 1: Evolution of $\bar{V}(\lambda)$ for the explicit (dash-dotted line) and the implicit schedulings (solid line)

For the initial condition $x_{0}=(-10,0)$, we choose $u_{0}=-B^{T} P\left(\lambda_{0}\right) x_{0}=0.57$ (with $\lambda_{0}$, the initial value of $\lambda$ for the implicit algorithm) which ensures that the implicit scheduling is initialized on the manifold $u+B^{T} P(\lambda) x=0$. As expected, we see that the initial $\lambda$ is larger for the implicit gain-scheduling, and that it increases faster. During the whole simulation, $\bar{V}=\bar{V}_{2}$ in the explicit scheduling, which means that the rate constraint is the limiting value. In the implicit scheduling, $\bar{V}=\bar{V}_{1}$ until $\lambda=0.81$, and $\bar{V}=\bar{V}_{2}$ afterwards, that is $u_{\max }$ is first the limiting value, and then $\dot{u}_{\max }$; this transition is especially visible in the change of slope at $\lambda=0.81$ on Figure 1 and in the first discontinuity in the $v$ graph in Figure 2. The second discontinuity is due to the interruption of the adaptation which eliminates the $B^{T} \frac{\partial P}{\partial \lambda} x \dot{\lambda}$ term in the expression of $v$. Figure 2 shows that the implicit scheduling allows for a higher peak for $x_{2}$, which accelerates the convergence of $x_{1}$ to the origin.

\section{Conclusion}

In this paper, we have presented a scheduling method that allows for the satisfaction of both stability and performance specifications for the control of linear systems subject to affine constraints. The Lyapunov-based scheduling 

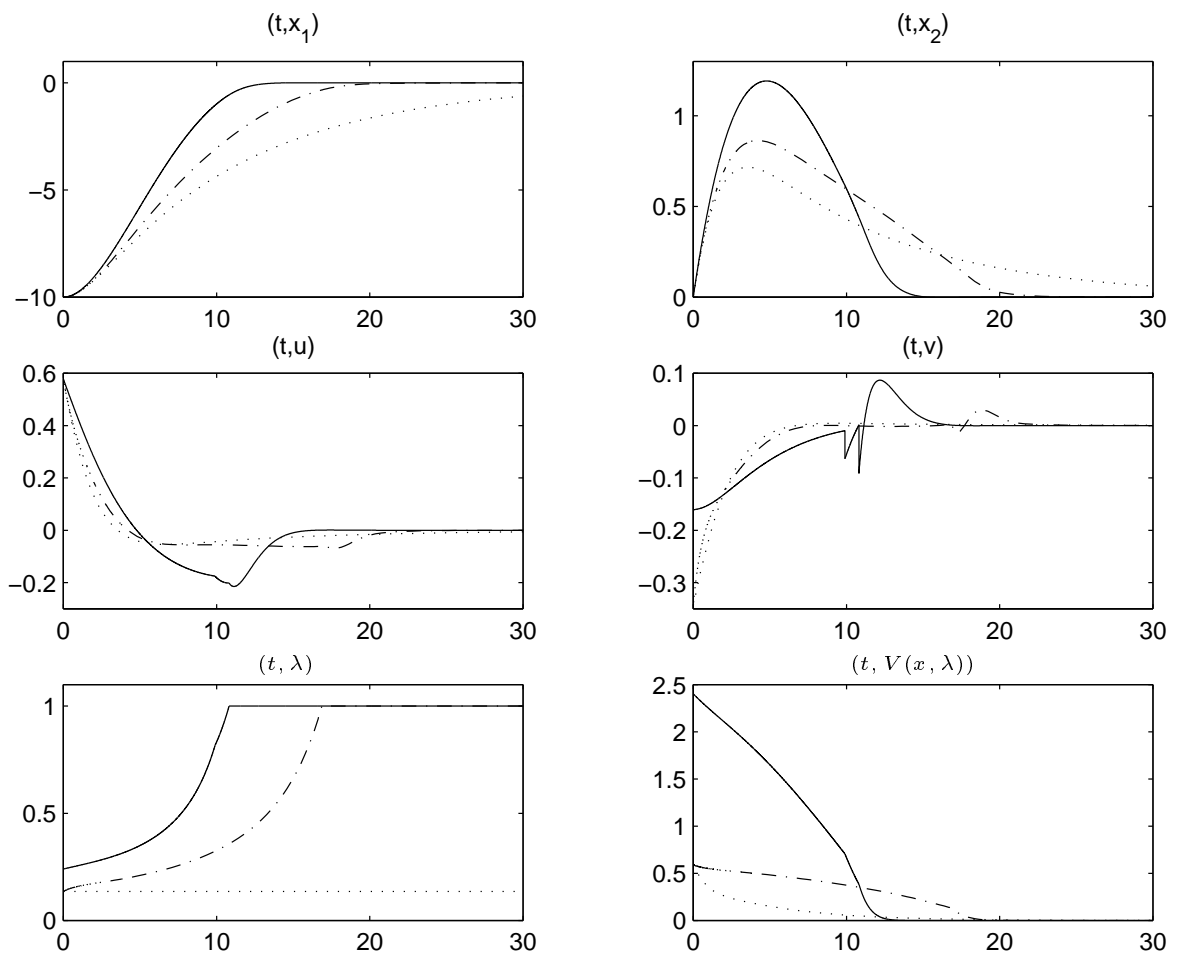

Figure 2: Solution of the controlled double integrator with amplitude and rate constraint on the control variable $\left(u_{\max }=1\right.$ and $\left.v_{\max }=1\right)$. The application of a low-gain control law without gain-scheduling (dotted line) is compared to a control law with explicit (dash-dotted line) and implicit gain-scheduling (solid line) 
provides online interpolation between an initial controller chosen from stability specifications and a target controller, chosen for local performance. For a given family of Lyapunov functions, two schedulings have been compared: an explicit gain-scheduling based on the control law $u=-K(\lambda) x$ and an implicit gain scheduling based on the invariance condition $K(\lambda) x=0$. The algorithms have been illustrated in the case of input magnitude and rate constraints.

\section{References}

[1] F. Grognard, R. Sepulchre, G. Bastin, "Improving the performance of low-gain designs for bounded control of linear systems", submitted to Automatica, CESAME Internal Report 99-52, Université catholique de Louvain, Belgium.

[2] Z. Lin. "Semi-global stabilization of linear systems with position and rate-limited actuators", Systems \& Control Letters, vol. 30, no. 1, pp. 1-11, 1997.

[3] Z. Lin, A.A. Stoorvogel, A. Saberi, "Output Regulation for Linear Systems Subject to Input Saturation", Automatica, vol 32, no. 1, pp. 29-47, 1996.

[4] A. Megretski, " $L_{2}$ BIBO output feedback stabilization with saturated control", Proceedings 13th IFAC World Congress, San Francisco, 1996, Vol D, pp. 435-440.

[5] R. Sepulchre, M. Jankovic, P.V. Kokotović, Constructive Nonlinear Control, Springer-Verlag, 1996.

[6] A. Stoorvogel, A. Saberi, "Output regulation of linear plants with actuators subject to amplitude and rate constraints"

[7] A. Teel, "Semiglobal stabilizability of linear null controllable systems with input nonlinearities", IEEE Trans. on Automatic Control, vol. 40, no. 1, pp. 96-100, 1995 